\title{
Um Sistema Multiagente para Recomendação de Conteúdos Educacionais para TV Digital Interativa
}

\author{
Raphael de Carvalho Muniz ${ }^{1,2}$, Rafael Castro de Sousa ${ }^{2}$, Francisco Milton \\ Mendes Neto ${ }^{1,2}$, Aquiles Medeiros Filgueira Burlamaqui ${ }^{3}$ \\ ${ }^{1}$ Programa de Pós-Graduação em Ciências da Computação - PPgCC \\ Universidade Federal Rural do Semi-Árido - UFERSA \\ BR 110 - Km 48, Bairro Pres. Costa e Silva CEP 59.610-090 Mossoró - RN \\ ${ }^{2}$ Núcleo Tecnológico de Engenharia de Software - NTES \\ Universidade Federal Rural do Semi-Árido - UFERSA \\ BR 110 - Km 48, Bairro Pres. Costa e Silva CEP 59.610-090 Mossoró - RN \\ ${ }^{3}$ Departamento de Informática e Matemática Aplicada \\ Universidade Federal do Rio Grande do Norte - UFRN \\ Bairro Lagoa Nova CEP 59.078-970 Natal - RN \\ $\begin{aligned} \text { raphaeldecm@gmail.com, } & \text { miltonmendes, rafaelcastro\}@ufersa.edu.br, } \\ & \text { aquiles@natalnet.br }\end{aligned}$
}

\begin{abstract}
The support of technological resources in teaching and learning has contributed to make them more efficient and enjoyable. The interactive digital $T V(i D T V)$ provides resources that make possible the development of a plethora of educational applications. However, an aggravating factor in the use of this media for education is show to users contents inadequate according their previous knowledge and other contextual information, like age, sex, region, personal interests, etc. This paper tries to fill in this gap by proposing an educational environment for iDTV, supported on standard of classification of T-SCORM learning objects, for recommending learning objects according to user context.
\end{abstract}

Resumo. $O$ apoio de recursos tecnológicos no processo de ensinoaprendizagem tem contribuído para torná-los mais eficientes e agradáveis. A $T V$ digital interativa (TVDi) provê recursos que tornam possível o desenvolvimento de uma gama de aplicações educacionais. No entanto, um fator agravante em seu uso na educação é exibir aos usuários conteúdos inadequados de acordo com o seu conhecimento anterior e outras informações contextuais, como idade, sexo, região, interesses, etc. Este artigo tenta preencher essa lacuna propondo um ambiente educacional para TVDi, apoiado no padrão de classificação de objetos de aprendizagem T-SCORM, para recomendação de objetos de aprendizagem de acordo com o contexto do usuário.

\section{Introdução}

Devido aos diferenciais que a TVDi possui, como sua aceitação pela comunidade, sua interatividade com os espectadores e ser um veículo de difusão de massa, é possível desenvolver uma série de aplicativos que podem ser integrados à programação de qualquer 
canal digital. Assim, devido as barreiras geográficas e financeiras enfrentadas pelos estudantes brasileiros, a TVDi surge como uma alternativa na oferta de educação de qualidade [MONTEZ and BECKER 2005].

O padrão para a classificação de OAs conhecido como SCORM (Shareable Content Object Reference Model), desenvolvido pela Advanced Distributed Learning (ADL), também é amplamente utilizado, e descreve como o conteúdo pode ser modelado e como os ambientes de gerenciamento de aprendizagem devem lidar com esse conteúdo para tornar viável sua reutilização [ADL 2010]. Com a proposta de tornar os OAs mais adequados por meios da sua especificação em SCORM, visando uma melhor apresentação na TVDi, foi desenvolvida uma extensão para esse padrão, chamada T-SCORM [SILVA 2012a], [SILVA et al. 2012]

Este trabalho propõe o desenvolvimento de um sistema multiagente capaz de recomendar OAs para TVDi de acordo com o contexto dos usuários, fazendo uso do padrão para a classificação de objetos de aprendizagem T-SCORM. A abordagem proposta neste trabalho utiliza um Algoritmo Genético (AG) para realizar a recomendação de OAs.

O trabalho está organizado da seguinte forma: A Seção 2 descreve a TV Digital Interativa. A Seção 3 apresenta conceitos sobre algoritmos genéticos. A Seção 4 descreve a abordagem proposta. A Seção 5 descreve o mecanismo de recomendação utilizado. E, por fim, a Seção 6 apresenta as considerações finais e os trabalhos futuros.

\section{TV Digital Interativa}

O surgimento de novas tecnologias para fins educacionais proporciona apoio ao processo de ensino-aprendizagem, tornando-o mais agradável, acessível e eficaz. Algumas tecnologias usadas antes para o entretenimento passaram a ser utilizadas para auxiliar a aprendizagem, como, por exemplo, o uso de animações e jogos interativos de apoio à aprendizagem [SANTOS 2007].

Outra tecnologia utilizada anteriormente apenas para o entretenimento e, atualmente, utilizada para fins educacionais é a TV, que hoje atinge cerca de $96 \%$ dos 49,8 milhões de domicílios no Brasil [IBGE 2010]. O processo de ensino e aprendizagem através TVDi é conhecido como t-learning ${ }^{1}$. Para fortalecer a inclusão digital no Brasil, uma das ações do governo brasileiro foi a criação do Decreto Presidencial No $4901 \mathrm{em}$ 26 de novembro de 2003, que fundou o Sistema Brasileiro de TV Digital (SBTVD). Apesar do trabalho realizado, ainda não há contribuições suficientes relacionadas com a concepção de novos produtos para TVDi [GOMES et al. 2008].

\section{Algoritmo Genético}

A recomendação de OAs apresentada neste artigo é realizada usando um Algoritmo Genético (AG), que pode ser definido como uma metáfora do processo biológico de evolução natural [LINDEN 2008]. AGs são responsáveis por tentar encontrar uma solução para os problemas que não têm algoritmos conhecidos [ARTERO 2009].

De acordo com [LINDEN 2008], a execução de um AG pode ser resumida nos seguintes passos: a) Inicializa-se a população de cromossomos; b) Avalia-se cada cromossomo da população; c) Seleciona-se os indivíduos que irão assumir o papel de pais

\footnotetext{
${ }^{1}$ Aprendizagem interativa a distância através da TV Digital [EDWIN et al. 2012]
} 
para gerar novos cromossomos; d) Aplica-se as operações de recombinação e mutação, a fim de selecionar os indivíduos como pais, criando uma nova geração; e) Elimina-se os cromossomos da geração anterior; f) Avalia-se os cromossomos que foram gerados e inseridos na população; g) Se os cromossomos encontrados representam a solução esperada para o problema ou o número máximo de gerações foi alcançado, ou o AG não conseguiu mostrar mais progresso, a execução é terminada. Caso contrário, a execução retorna para o passo c.

\section{Abordagem proposta}

$\mathrm{O}$ ambiente desenvolvido consiste em um sistema multiagente que conta com quatro agentes. O Agente Estudante é responsável por monitorar as atividades dos alunos no Moodle ${ }^{2}$ e enviar informações sobre o perfil da turma para o Agente de Conteúdo. Os alunos fornecem essas informações durante a inscrição no curso, através do formulário de cadastro do Moodle. O primeiro OA recomendado será compatível com o nível de conhecimento fornecido pela turma em seu perfil estático, enquanto que o nível do próximo OA dependerá da interação posterior entre os alunos e o OA recomendado anteriormente, o que será alcançado através das atividades desenvolvidas no final de cada OA.

O Agente de Conteúdo, ao receber as informações sobre o perfil da turma, é encarregado de executar o algoritmo genético. Informações relacionadas ao perfil da turma são recuperadas tanto da ontologia de contexto estático, que é alimentada no momento do registro no curso com informações que os usuários fornecem, quanto da ontologia do contexto dinâmico, que é alimentada a partir de interações dos usuários no sistema. Ao término da execução do AG, o agente de conteúdo envia as informação dos OAs que serão exibidos na TVDi para o Agente de Interface, o qual é responsável por apresentar os OAs de acordo com as características de cada OA.

Ao iniciar o sistema, todos os agentes devem registrar os serviços que cada um oferece no Agente DF, que é responsável por informar aos outros agentes que serviços estão disponíveis e qual agente é capaz de executá-lo. Este processo consiste no serviço de páginas amarelas.

\section{Mecanismo de Recomendação}

Para efetuar uma recomendação mais adequada de OAs por meio de algoritmo genético, foram levadas em consideração características relevantes no processo de ensinoaprendizagem. Estas características são descritas a seguir.

\subsection{Nível de Conhecimento}

No ato da inscrição para um curso, os alunos preenchem um formulário com suas informações pessoais. Um dos dados apresentados é o nível de conhecimento que o aluno considera ter em relação ao curso que está inscrito. O grau de conhecimento do grupo de usuários pode ser determinado por meio de uma média aritmética simples. Nos metadados de cada OA existe uma variável que determina o nível de dificuldade do OA. Esta dificuldade pode variar entre fácil, médio ou difícil. Para calcular o grau de compatibilidade dos OAs com o curso, foi levado em consideração o grau de relevância entre o nível de conhecimento do grupo e o grau de dificuldade do OA, variando de 0 (não adequado) a 5 (totalmente adequado).

\footnotetext{
${ }^{2}$ Ambiente de Aprendizagem Moodle. http://www.moodle.org.br/
} 


\subsection{Afinidade com o curso}

Dependendo do repositório de OAs utilizado para a aplicação de um curso, é provável que haja vários OAs que não são adequados para serem recomendados de acordo com o conteúdo do curso. Então, é importante definir o grau de afinidade dos OAs com o curso. Esta variável determina o grau de afinidade do conteúdo do OA com o curso, que pode variar de 0 (nenhuma afinidade) até 5 (alta afinidade).

\subsection{Palavras-chave}

Os OAs presentes no repositório estão no padrão T-SCORM, que mostra, em seus metadados, palavras-chave relevantes sobre o assunto do OA. Por meio dessas palavras-chave, é possível verificar o nível de afinidade do OA com o curso. O nível de afinidade é dado por uma escala em que é atribuído um peso 5 (cinco) para o OA com maior incidência de palavras-chave relacionadas ao curso e pesos proporcionalmente menos para os outros OAs presentes no repositório.

\subsection{Representação do Cromossomo}

Neste trabalho optou-se por utilizar a representação binária, pois proporciona uma melhor utilização dos recursos de processamento, fácil e eficiente manipulação de seus operadores genéticos no cromossomo [PETROLI NETO 2011].

Cada indivíduo da população (cromossomo) é composto de 51 bits. Cada OA é representado por 17 bits, em que os primeiros dez bits são usados para identificação (ID) do OA e os últimos sete bits representam a sua aptidão, de modo que cada cromossomo possui três OAs. A identificação do OA corresponde à posição do objeto na tabela no banco de dados do Moodle e sua aptidão corresponde à soma das características mencionadas nas subseções anteriores.

\subsection{Aplicando o Algoritmo Genético}

A população inicial foi selecionada aleatoriamente, pois existem muitos trabalhos sobre AG que mostram que a geração da população inicial não é uma fase crítica [ZINI 2009]. O mecanismo utilizado para efetuar a seleção dos pais foi a seleção da roleta que, segundo [LINDEN 2008], é o método mais utilizado para este fim. Este método funciona através da criação de uma roleta virtual em que cada indivíduo recebe uma porção da roleta proporcional à sua função de aptidão. Em seguida, a roleta é rodada e o indivíduo referente a porção em que a roleta parou é selecionado.

Após a seleção dos pais para reprodução, é utilizado o operador de cruzamento genético para gerar os próximos indivíduos da população, o que pode gerar melhores indivíduos ou não. Este processo é repetido várias vezes até chegar a um resultado satisfatório [ZINI 2009]. Neste processo, os bits para a esquerda do corte1 do pai1 são enviados para o primeiro filho e os bits entre o primeiro e segundo corte do mesmo pai são enviados para o segundo filho. Os bits após o segundo corte do pail permanecem no filho1. O segundo filho é formado pelos bits restantes [SILVA 2012b].

\section{Considerações Finais}

Neste artigo, foi apresentada uma abordagem baseada em agentes para a recomendação de OAs apoiada pelo padrão de classificação T-SCORM. A abordagem proposta tem a finali- 
dade de tornar o ambiente de aprendizagem adequado às necessidades de cada aluno, considerando o ambiente no qual ele está inserido. Foram realizados alguns testes do mecanismo de recomendação e os resultados obtidos foram considerados satisfatórios. Em 5 testes de recomendação realizados, foram recomendados 5 cromossomos e recomendados 15 OAs. Dos 15 OAs recomendados, os resultados obtidos foram: 10 OAs fortemente relacionados à disciplina, 2 OAs fortemente relacionados ao curso e 3 OAs não relevantes ao curso. O repositório de OAs utilizado nos testes continha a mesma proporção de OAs relevantes para a disciplina, OAs relevantes para o curso e OAs não relevante para o curso.

\section{References}

ADL (2010). "Advanced distributed learning". Disponível em: http://www.adlnet.org.

ARTERO, A. O. (2009). "Inteligência Artificial - Teoria e Prática". São Paulo: Livraria da Física, 1. ed.

CARNEIRO, N., MARQUES, A., TEIXEIRA, R., MEIGUINS, A., and MEIGUINS, B. (2012). "Design decisions for a Brazilian t-commerce application. Information Visualisation (IV)". 2 ed. 16th International Conference on, Montpellier. p. 176-181.

EDWIN, M., CARLOS, M. J., JUAN, T., CESAR, R. J., JOHAN, V. M., and OSCAR, I. T. (2012). "Multiplatform learning system based on interactive digital television technologies, IDTV". Informatica (CLEI), 2012 XXXVIII Conferencia Latinoamericana En, Medellin. p. 1-10.

GOMES, A. S., MONTEIRO, B., MELO, C., ARCOVERDE, D., and FROTA, C. (2008). "Design da Interação de Novos Produtos para TVD: Abordagens qualitativas". IHC, Porto Alegre, RS.

IBGE (2010). "Instituto Brasileiro de Geografia e Estatística". Disponível em: http://www.ibge.gov.br/home/default.php.

LINDEN, R. (2008). "Algoritmos Geneticos - Uma importante ferramenta da Inteligencia Computacional". 2 ed. Rio de Janeiro, RJ: Brasport.

MONTEZ, C. and BECKER, V. (2005). "TV Digital Interativa: Conceitos, desafios e perspectivas para o Brasil". Florianópolis: Editora da UFSC, 2 ed.

PETROLI NETO, S. (2011). "Computação Evolutiva: desvendando os algoritmos genéticos". Ubiquidade - Revista de Estudos de Tecnologia da Informação e Comunicações. v. 1, n. 1, p. 34-35.

SANTOS, D. T. (2007). "Estudo de aplicativos de TVDi para educação a distância". 98p. Dissertação (Mestrado em Engenharia Elétrica) - UNICAMP, Campinas, SP.

SILVA, F. M. (2012a). "T-SCORM: Uma Extensão do Padrão SCORM para Apoiar o Projeto de Conteúdos Educacionais para t-learning”. 75p. Dissertação (Mestrado em Ciências da Computação) - Universidade Federal Rural do Semi Arido, Mossoró - RN.

SILVA, F. M., MENDES NETO, F. M., BURLAMARQUE, A. M. F., and DUARTE, D. H. (2012). "TSCORM: An Extension of the SCORM Standard to Support the Project of Educational Contents for t-learning". Creative Education, v. 03, p. 101-108.

SILVA, L. C. N. (2012b). "MobiLE: Um Ambiente Multiagente de Aprendizagem Móvel para Apoiar a Recomendação Ubíqua de Objetos de Aprendizagem”. 107 p. Dissertação (Mestrado em Ciências da Computação) - Universidade Federal Rural do Semi Arido, Mossoró-RN.

ZINI, E. O. C. (2009). "Algoritmo Genético Especializado na Resolução de Problemas com Variáveis Contínuas e Altamente Restritos”. 149p. Dissertação (Mestrado em Engenharia Elétrica) - Universidade Estadual Paulista (UNESP). Ilha Solteira, SP. 